CASE REPORT

\author{
R.D. Murtagh \\ M.J.D. Post \\ J. Bruce \\ K.K. Post
}

\section{Spinal Epidural Aspergillosis in a Patient With HIV Resulting From Long-Standing (3 Years) Lung Infection}

\begin{abstract}
SUMmaRY: We present an unusual case of a man with human immunodeficiency virus (HIV) with pulmonary aspergillosis and spinal invasion and compression of the spinal cord occurring during a long period (3 years), as documented by MR imaging and surgical intervention. Invasive pulmonary aspergillosis with cord compression has been reported in the past, but, to the best of our knowledge, none of these have been in a patient with HIV.
\end{abstract}

$\mathbf{H}$ ost defense against the Aspergillus species is considered to be a function of neutrophils. As such, invasive aspergillosis is relatively uncommon in human immunodeficiency virus (HIV), a disorder of lymphocyte function. We describe a case in which a man with well-controlled HIV presented with invasive pulmonary aspergillosis with cord compression. We will provide several reasons why invasive aspergillosis is thought to occur in patients with HIV, as well as describe the MR imaging features of this case, which can serve as important diagnostic clues.

\section{Case Report}

A 39-year-old man was diagnosed in 1997 with HIV. He was started on stavudine (Zerit) and lopinavir and ritonavir (Kaletra) with good control of his disease. In May 2003, his white blood cell (WBC) count was 5800 cells/uL with $58 \%$ neutrophils (reference range $>34 \%$ ). CD4 count was 507 (reference range $>400$ cells/uL) with a quantitative serum HIV count of less than 20.

In September 2003, he presented with cough and chest pain. Plain films and chest CT revealed a right upper lobe mass without definite chest wall or mediastinal invasion. He had a WBC count of 9200 with only $5.8 \%$ neutrophils and an absolute neutrophil count (ANC) of 0.5 (normal >1.6). Results of 2 CT-guided percutaneous biopsies revealed only chronic inflammation and fibrosis. Results of an open biopsy in January 2004 revealed fibrosis and "ribbonlike" fungal elements with occasional septations, for which he was given intravenous amphotericin B and, later, oral voriconazole for presumed aspergillosis. His WBC count was 6300 with $40 \%$ neutrophils and an ANC of 2.8 .

The patient remained on antifungal therapy for the next $2 \frac{1}{2}$ years. During this time, he had a stable chest CT, WBC, and neutrophil and $\mathrm{CD} 4$ counts. MR imaging showed an isointense to hypointense mass on T2-weighted images, with invasion into the chest wall and mediastinum, extension into the right $\mathrm{T} 1-\mathrm{T} 2$ and $\mathrm{T} 2-\mathrm{T} 3$ neural foramina, subtle bony involvement, and minimal ventral epidural spread. Lost to follow-up, the patient presented 7 months later to the emergency department with severe right shoulder and thoracic back pain, as well as weakness in the bilateral lower extremities. Thoracic MR imaging revealed invasion into the $\mathrm{T} 1-\mathrm{T} 3$ vertebral bodies and posterior ele-

Received June 20, 2007; accepted June 28

From the Departments of Radiology (R.D.M., M.J.D.P.), and Pathology (J.B.), University of Miami Miller School of Medicine, Miami, Fla; and the University of Miami Miller School of Medicine (K.K.P.), Miami, Fla.

Please address correspondence to Ryan D. Murtagh, MD, MBA, University Diagnostic Institute, 3301 Alumni Drive, Tampa, FL 33612.

DOl 10.3174/ajnr.A0782 ments, widening of the right T1-T2 through T3-T4 neural foramina, and epidural extension with severe cord compression from $\mathrm{T} 1$ to $\mathrm{T} 3$. Again, the signal intensity of this soft tissue mass was characterized by isointensity to hypointensity on T2-weighted images with heterogeneous enhancement after administration of contrast (Figs 1 and 2). An emergency decompressive laminectomy of T1-T3 was performed with subtotal resection of the mass. Pathologic examination revealed large amounts of attenuated connective tissue with acute and chronic inflammation as well as fungal organisms with pseudohyphae. Staining confirmed aspergillosis; culture medium grew the Aspergillus fumigatus species (Fig 3).

\section{Discussion}

Invasive aspergillosis has not traditionally been considered a complication of well-controlled HIV infection. ${ }^{1}$ Host defense against the Aspergillus species is postulated to be a function of neutrophils. ${ }^{2}$ As such, the frequency of Aspergillus infection is increased in patients with neutropenia, such as those with acute leukemia, or in those with abnormal neutrophil function, such as is seen in chronic granulomatous disease. ${ }^{1-3} \mathrm{~Pa}-$ tients being treated with corticosteroids, such as transplant recipients, are also susceptible because these drugs potentiate risk for Aspergillus infection by decreasing intracellular killing of spores by macrophages, thereby allowing intracellular germination. ${ }^{4}$

HIV, on the other hand, is an infection of lymphocytes and, in isolation, should not theoretically place the patient at a higher risk for colonization and infection. ${ }^{2}$ However, studies have shown a direct relationship between severe HIV infection and neutropenia. ${ }^{1,5}$ Other possible explanations for infection in patients with HIV disease include the increasing use of corticosteroid treatment, as is used in the treatment of Pneumocystis carinii pneumonia ${ }^{1,6,7}$; neutropenia-inducing antiretroviral drugs such as zidovudine $e^{1,6}$; and a prolonged life of patients with late-stage HIV disease. ${ }^{1,6,7}$

We find 3 interesting features of this case. First, direct spinal invasion by pulmonary aspergillosis, though rare, is not unheard of, as several cases of chest wall invasion with epidural aspergillosis have been documented in the past. ${ }^{3,4,8-11}$ As far as we know, however, there has been only 1 reported case of epidural aspergillosis in a patient with HIV disease, and that patient was not found to have pulmonary involvement. ${ }^{2}$

Second, the initial presentation and diagnosis of pulmonary aspergillosis in this patient correlates with a relatively acute onset of neutropenia. Our patient maintained a normal neutrophil count between 1997 and early 2003 and, during 

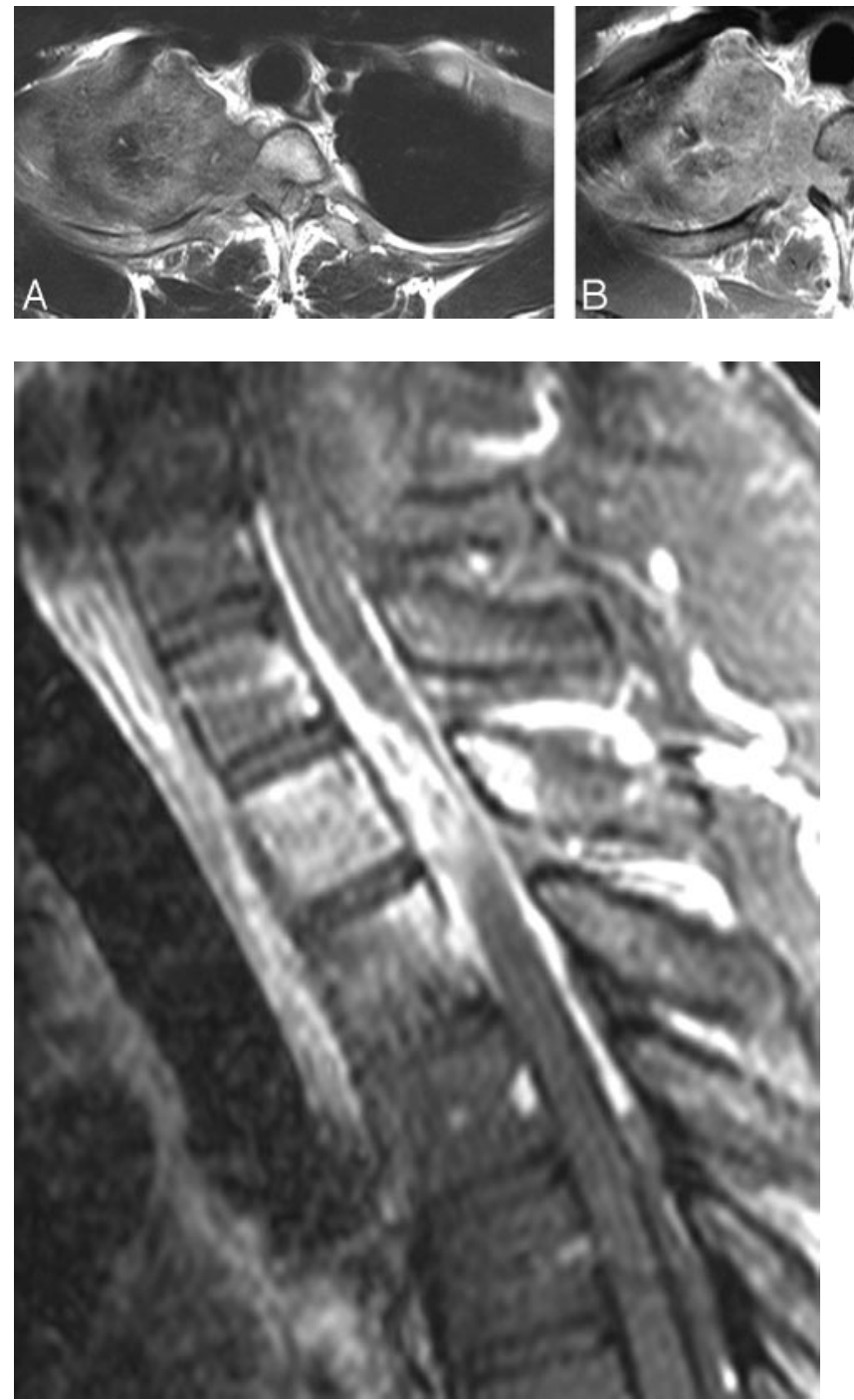

Fig 2. Sagittal postgadolinium, fat-saturated image demonstrates avid enhancement of the mass, the epidural space circumferentially, and the T1-T3 vertebral bodies.

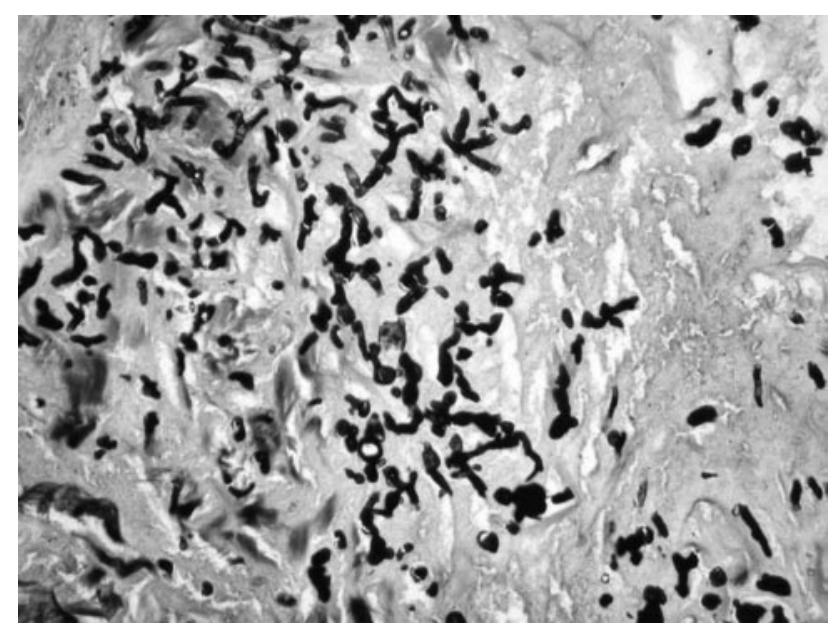

Fig 3. Gomori methenamine silver (GMS) stain for fungus showing branching hyphae. The fungal elements stain black (silver).

that period, was asymptomatic. At the time of initial presentation, however, he was found to have significant neutropenia with only $5.8 \%$ neutrophils and an ANC of 0.5 (normal >1.6).
This finding correlates with the hypothesis that host defense against the Aspergillus species is at least, in part, because of neutrophil function. It is not known why the patient became neutropenic, and it is not fully understood why the patient had progression of disease despite subsequent maintenance of normal neutrophil counts, treatment with appropriate antifungal therapy, and maintenance of CD4 counts well above 200.

Finally, the presence of isointensity to low intensity on T2weighted images in the lesion is of interest. A study by Herold et $\mathrm{al}^{12}$ described the "targetlike" appearance of pulmonary nodular infiltrates, with low signal intensity centrally and increased intensity peripherally on both T1- and T2-weighted images. The central low signal intensity was attributed to both central cavitation and coagulative fungal necrosis, whereas the rim of increased signal intensity was attributed to subacute hemorrhage or hemorrhagic infarction. A study by Kawashima et $\mathrm{al}^{3}$ reported MR imaging findings in 2 patients with chronic granulomatous disease, pulmonary aspergillosis, and invasion into the chest wall. In both cases, the lesions were increased in T2 signal intensity. Finally, a case report by Fujimoto et $\mathrm{al}^{13}$ described low T2 signal intensity of an aspergilloma located within a cavitary lung neoplasm that showed high T2 signal intensity. In our patient, the areas of pulmonary consolidation and invasion into the chest wall were uniformly low or isointense on T2-weighted images. On pathologic examination, the resected epidural mass was found to have large amounts of attenuated connective tissue with areas of both acute and chronic inflammation. We postulate that good control of HIV in this patient allowed for production of an effective immune response, thereby producing chronic inflammatory changes and fibrosis. The fibrosis, in turn, may account for the decreased T2 signal intensity. Furthermore, the presence of paramagnetic and ferromagnetic elements intrinsic to the fungi may contribute to the T2 hypointensity. ${ }^{14}$

In summary, invasive aspergillosis is felt to have a generally poor outcome. As such, early diagnosis and treatment are imperative to survival. The radiologist should be aware of the imaging characteristics and clinical presentation of invasive aspergillosis in patients with HIV. We conclude that epidural aspergillosis should be considered even in the setting of wellcontrolled HIV and that the isointense to hypointense signals on T2-weighted images are a helpful diagnostic clue.

\section{References}

1. Wallace JM, Lim R, Browdy BL, et al. Risk factors and outcomes associated with identification of Aspergillus in respiratory specimens from persons with HIV disease. Pulmonary Complications of HIV Infection Study Group. Chest 1998;114:131-37

2. Go BM, Ziring DJ, Kountz DS. Spinal epidural abscess due to Aspergillus sp in a patient with acquired immunodeficiency syndrome. South Med J 1993;86:957-60

3. Kawashima A, Kuhlman JE, Fishman EK, et al. Pulmonary Aspergillus chest 
wall involvement in chronic granulomatous disease: CT and MRI findings. Skeletal Radiol 1991;20:487-93

4. Wagner DK, Varkey B, Sheth NK, et al. Epidural abscess, vertebral destruction, and paraplegia caused by extending infection from an aspergilloma. Am JMed 1985;78:518-22

5. Levine AM, Karim R, Mack W, et al. Neutropenia in human immunodeficiency virus infection: data from the women's interagency HIV study. Arch Intern Med 2006;166:405-10

6. Minamoto GY, Barlam TF, Vander Els NJ. Invasive aspergillosis in patients with AIDS. Clin Infect Dis 1992;14:66-74

7. Denning DW, Follansbee SE, Scolaro M, et al. Pulmonary aspergillosis in the acquired immunodeficiency syndrome. N Engl J Med 1991;324:654-62

8. Seres JL, Ono H, Benner EJ. Aspergillosis presenting as spinal cord compression. Case report. J Neurosurg 1972;36:221-24
9. Polatty RC, Cooper KR, Kerkering TM. Spinal cord compression due to an aspergilloma. South Med J 1984;77:645-48

10. Dietz R, Huber G, Thetter O, et al. Aspergillosis of the lung with osteoclasis and paraplegia. Neuroradiology 1982;23:219-21

11. Hendrix WC, Arruda LK, Platts-Mills TA, et al. Aspergillus epidural abscess and cord compression in a patient with aspergilloma and empyema. Survival and response to high dose systemic amphotericin therapy. Am Rev Respir Dis 1992;145:1483-86

12. Herold CJ, Kramer J, Sertl K, et al. Invasive pulmonary aspergillosis: evaluation with MR imaging. Radiology 1989;173:717-21

13. Fujimoto K, Meno S, Nishimura $\mathrm{H}$, et al. Aspergilloma within cavitary lung cancer: MR imaging findings. AJR Am J Roentgenol 1994;163:565-67

14. Zinreich SJ, Kennedy DW, Malat J, et al. Fungal sinusitis: diagnosis with CT and MR imaging. Radiology 1988;169:439-44 